Günter Herfert

\title{
Disurbanisierung und Reurbanisierung
}

Polarisierte Raumentwicklung in der ostdeutschen Schrumpfungslandschaft*

\section{Disurbanisation and Reurbanisation}

\author{
Polarised spatial development in the "shrinking" landscape of eastern Germany
}

\begin{abstract}
Kurzfassung
Die Raumentwicklung der 90er Jahre wurde in Ostdeutschland wesentlich durch kleinräumige Suburbanisierungsprozesse geprägt; nunmehr deutet sich ein Wechsel zur großräumigen Disurbanisierung mit vereinzelten Reurbanisierungstendenzen an. Gleichzeitig entstehen neue polarisierte Entwicklungsmuster. Abgesehen von wenigen Stabilitätsinseln in einer ansonsten demographischen Schrumpfungslandschaft bilden sich räumliche Cluster mit überdurchschnittlichen Bevölkerungsverlusten, hervorgerufen durch Sterbefallüberschüsse und den Wegfall dominanterWanderungsströme. Verlierer der aktuellen Entwicklung sind insbesondere die Städte. Auf Grund der wirtschaftlichen Rahmenbedingungen ist im Osten Deutschlands mit Ausnahme weniger Reurbanisierungsinseln wie u.a. die Regionen Leipzig, Dresden und die thüringische Städtereihe weitflächig mit einem anhaltenden Disurbanisierungsprozess zu rechnen.
\end{abstract}

\begin{abstract}
Spatial development in eastern Germany in the '90s was marked to a very significant degree by processes of relatively small-scale suburbanisation; there are now clear signs of a change towards processes of large-scale disurbanisation with occasional tendencies towards reurbanisation. At the same time, new polarised development patterns are emerging. If we disregard a small number of islands of stability in a demographic landscape otherwise characterised by shrinkage, the pattern emerging is one of spatial clusters with above-average levels of population depletion, attributable both to natural wastage (more deaths than births) and to the absence of major migration streams. In the context of current developments, the losers are in particular the towns and cities. In view of the prevailing economic climate in eastern Germany, disurbanisation processes can be expected to continue unabated over large areas of the territory, with exceptions to this trend only in a few islands of reubanisation, such as the Leipzig and Dresden regions and the city-axis found in Thuringia.
\end{abstract}

Die 1990er Jahre waren in den neuen Ländern durch eine sehr dynamische Stadt-Umland-Wanderung geprägt (Aring u. Herfert 2001; Herfert 2001). Mit Beginn des neuen Jahrhunderts zeigen sich in den Stadtregionen Anzeichen für einen Wechsel von der Suburbanisierungs $^{-1}$ in die Disurbanisierungs- und teilweise auch Reurbanisierungsphase, eingebettet in einen anhaltenden demographischen Schrumpfungsprozess.
Nach einer kurzzeitigen, von 1993-1998 andauernden Dekonzentrationsphase der Bevölkerung in den Stadtregionen dominieren zunehmend wieder die kleinräumigen zentripetalen Wanderungsströme, sind wieder wie vor der Wende - Konzentrationsprozesse im Siedlungsnetz zu beobachten. Eine Großstadt wie Leipzig, die 1997 im Saldo noch über 12000 Personen an ihr Umland verlor, wird schätzungsweise Ende 2002 
wieder leichte Wanderungsgewinne aus dem Umland und insgesamt eine leichte Bevölkerungszunahme verzeichnen. Im suburbanen Raum hat hingegen bereits ein Schrumpfungsprozess eingesetzt. ${ }^{2}$ Es scheint, dass sich innerhalb kürzester Zeit das Muster der Raumentwicklung in Ostdeutschland wieder grundlegend verändert und sich zunehmend regional stärker differenziert. Unter diesen Bedingungen stellt sich die Frage, welche neuen Räume demographischer Stabilität bzw. zunehmender Instabilität sich ausprägen. Grundsätzlich geht man davon aus, dass es vorrangig die strukturschwachen Räume sein werden, die von einem verstärkten Bevölkerungsrückgang betroffen werden. Darüber hinaus ist es für neue Planungsstrategien jedoch wichtig zu wissen, wo und wie diese Schrumpfungsprozesse ablaufen. Dementsprechend beleuchtet der Beitrag den aktuellen Wandel demographischer Raummuster beispielhaft in den Bundesländern Sachsen, Sachsen-Anhalt und Thüringen und versucht, Problemzonen der räumlichen Entwicklung in diesen drei Bundesländern aufzudecken.

\section{Rückblick auf die 1990er Jahre}

In den neuen Ländern wurde - nach der Westdrift von 1989 bis 1991 - die Bevölkerungsentwicklung bis Mitte der zweiten Hälfte der 1990er Jahre im Wesentlichen durch die Wohnsuburbanisierung geprägt. Mehr oder weniger geschlossene ringförmige Wachstumsräume um Groß-, Mittel- und teilweise sogar auch Kleinstädte in einem ansonsten demographisch schrumpfenden Raum waren das typische Raummuster in Ostdeutschland (Abb. 1). Die Wohnsuburbanisierung wurde zu einem fast ubiquitären Phänomen: Sie wurde, und darüber besteht heute in Wissenschaft und Politik ein breiter Konsens, wesentlich - neben weiteren Faktoren (s.a. Aring u. Herfert 2001) - durch Umfang und Dauer des Fördergebietsgesetzes Ost sowie weitere Subventionierungen im Wohnungsbau - ohne jegliche räumliche Steuerungskomponente - geprägt und „künstlich“ forciert (Herfert 1998). Diese wohnungspolitischen Fördermaßnahmen ließen eine Differenzierung der Regionalentwicklung hinsichtlich regionaler Entwicklungspotenziale in den Hintergrund treten. In Anbetracht der entstandenen hohen Wohnungsleerstände in Ostdeutschland und der riesigen Kosten, die für den Stadtumbau Ost zu erwarten sind, kann diese Entwicklung in Anlehnung an Conrad (1980) im Nachhinein als „volkswirtschaftlich in höchstem Maße verschwenderisch “` bezeichnet werden.

Die Gleichartigkeit der interregionalen Raumentwicklung bis in die zweite Hälfte der 90er Jahre spiegelt sich auch im Vergleich der Gemeindengrößengruppen nach

\section{Abbildung 1}

Wanderungssalden der Umlandgemeinden von Oberzentren in Sachsen, Sachsen-Anhalt und Thüringen 1993-1998

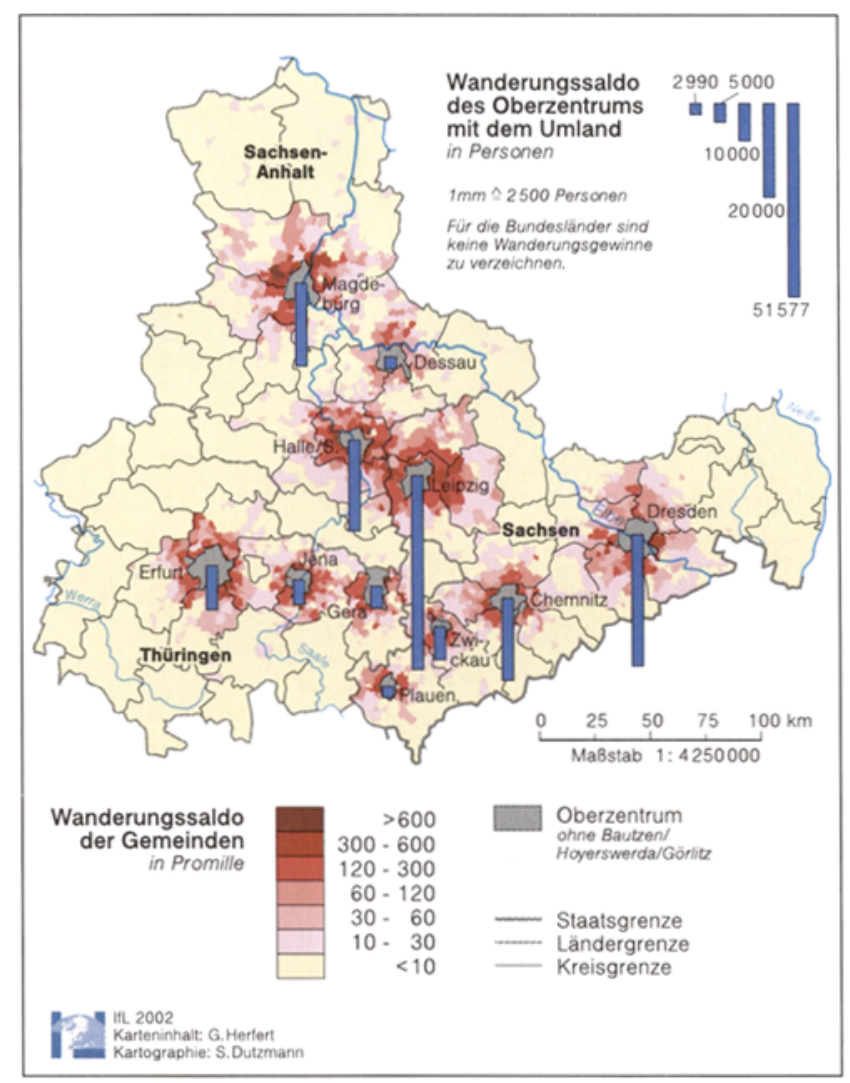

Quelle: Herfert 2001, geändert

siedlungsstrukturellen Raumtypen ${ }^{4}$ wider (Abb. 3). Generell zeigt sich das gleiche Muster: wachsende Gemeinden bis 2000 Einwohner auf der einen Seite bedingt durch den dynamischen Wohnsuburbanisierungsprozess - und Bevölkerungsverluste der Städte, insbesondere der Mittel- und Großstädte, auf der anderen Seite.

\section{Die Trendwende}

Ende der 90er Jahre änderte sich dieses Verteilungsmuster grundlegend. Wenngleich insbesondere im engeren Umland der Kernstädte noch einzelne Gemeinden ein leichtes Bevölkerungswachstum hatten (Abb. 2), stellte sich jetzt auch in den unteren Gemeindegrößengruppen ein negativer Entwicklungstrend ein (Abb. 3). Aktuelle Daten zur Stadtregion Leipzig - eines der prägnantesten Beispiele ostdeutscher Suburbanisierung - zeigen, dass auch im suburbanen Raum die demographische Schrumpfung dominiert, da die Stadt-Umland-Wanderung ihre Funktion als entscheidende Komponente der Bevölkerungsbewegung ver- 
Abbildung 2

Durchschnittliche jährliche Bevölkerungsentwicklung der Gemeinden in Sachsen, Sachsen-Anhalt und Thüringen 1990-1998 und 1998-2000

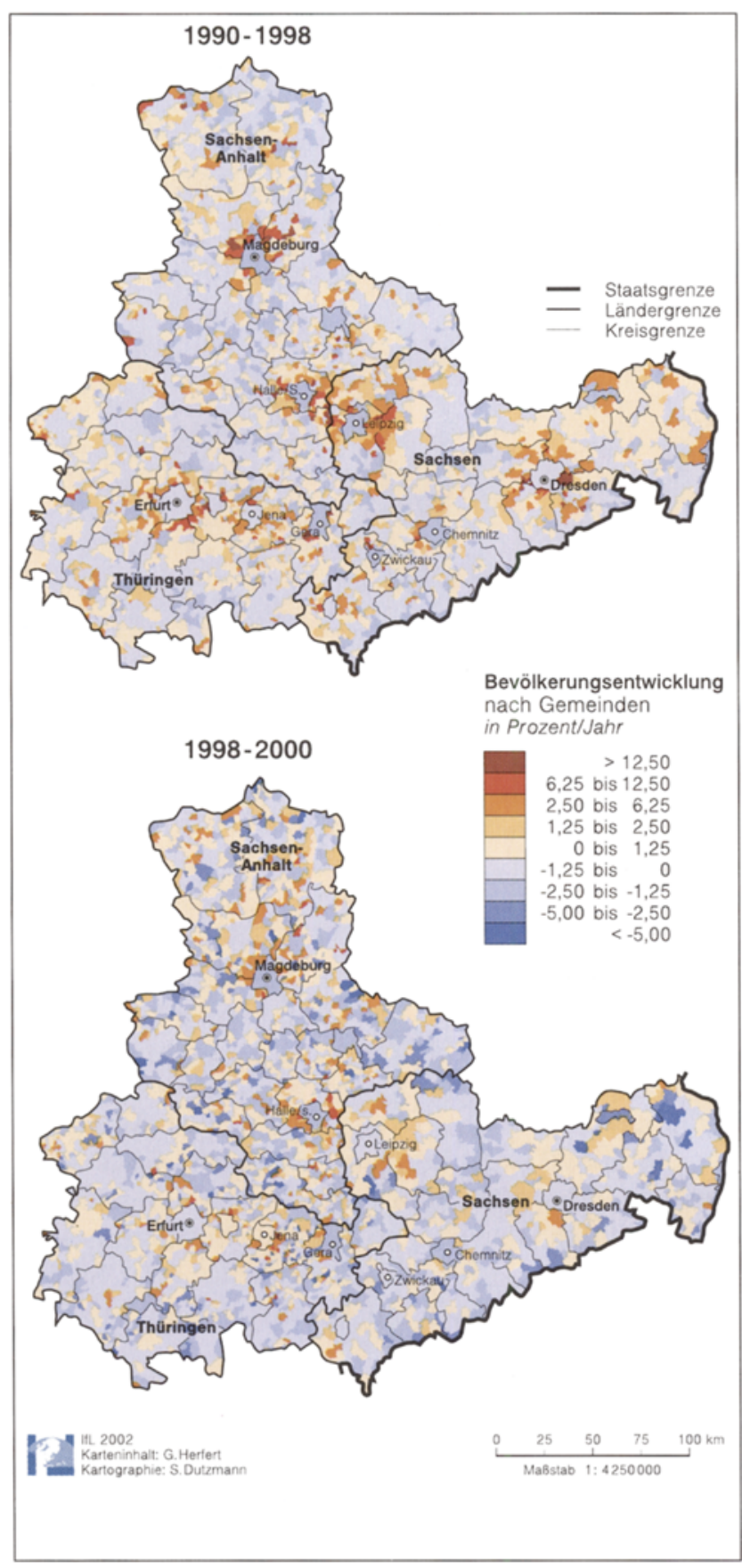

Quelle: Herfert u. Schulz 2002, ergänzt

loren hat. So verzeichnete der Kreis Leipziger Land gegenüber der Stadt Leipzig Ende 2001 nur noch einen sehr geringen Wanderungsgewinn (373 Personen). Mit Ausnahme einiger Kleinstädte in unmittelbarer Randlage zu Leipzig waren die Wanderungsbilanzen der suburbanen Gemeinden in der Regel ausgeglichen bis deutlich negativ.

\section{Abbildung 3}

Durchschnittliche jährliche Bevölkerungsentwicklung von Gemeindegrößengruppen nach Regionstypen in Sachsen, Sachsen-Anhalt und Thüringen 1990-1994, 1994-1998 und 1998-2000

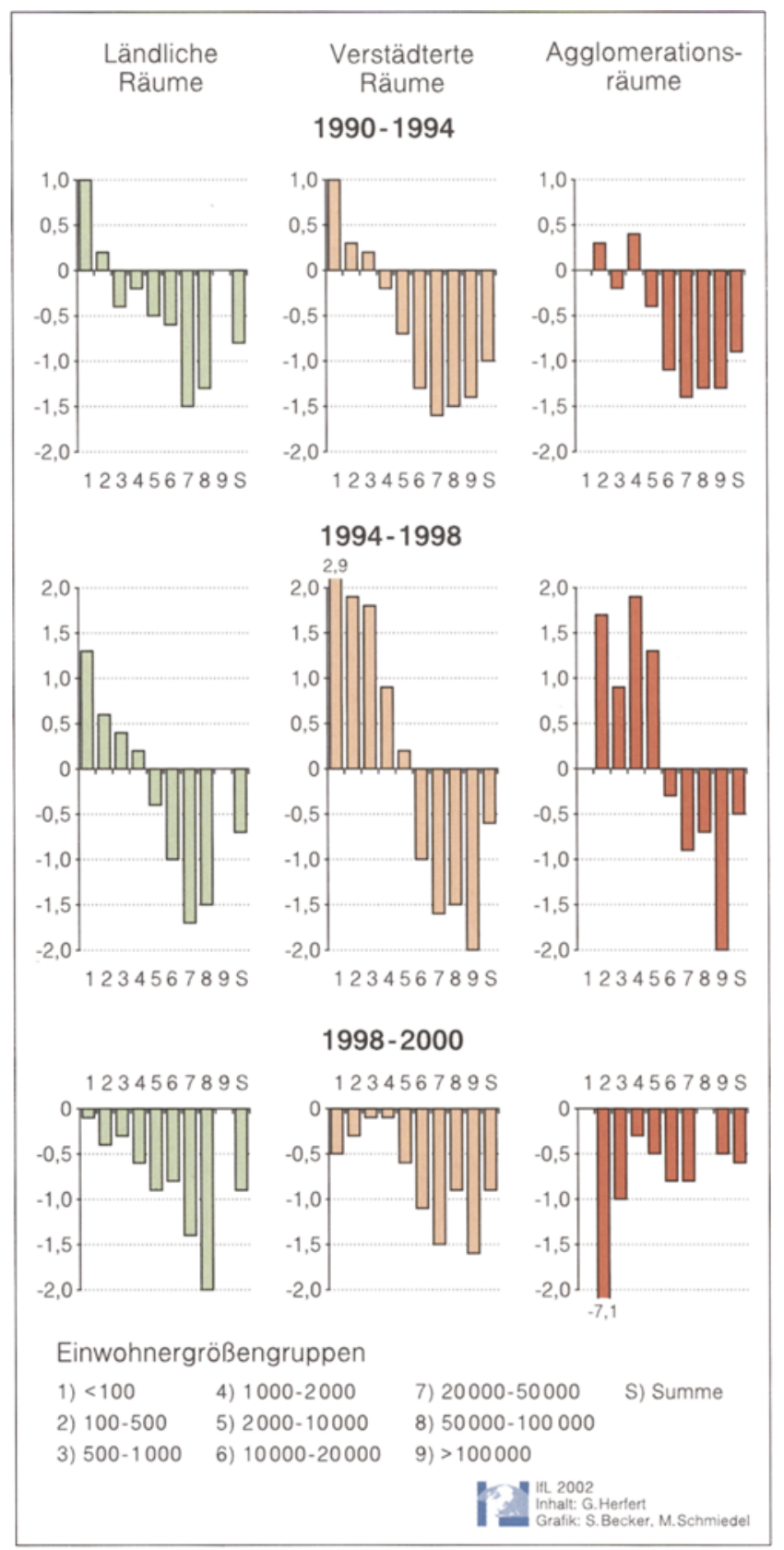

Quelle: Statistische Landesämter, eigene Berechnungen

Noch Mitte der 90er Jahre gingen Prognosen von einem zeitweiligen Rückgang der Wohnsuburbanisierung aus, jedoch nicht von einer Umkehr dieses Prozesses. Ursache für diese Trendumkehr war nicht nur der Wegfall der exorbitant hohen steuerlichen Sonderabschreibungen in den neuen Ländern - dieser erfolgte zu einem Zeitpunkt, wo u. a. die Nachfrage nach suburba- 
nen Mietwohnungen bereits weggebrochen war. Ein wesentlicher Faktor war hingegen die zunehmende Attraktivität urbanen Wohnens infolge umfangreicher Modernisierungsmaßnahmen, eines breit gefächerten Wohnungsangebotes und eines niedrigen Mietpreisniveaus in der Kernstadt, was letztlich das Preisgefälle zum Umland so gut wie auflöste. Insbesondere die gehobenen gründerzeitlichen Quartiere der Kernstädte wurden zum Zielgebiet innerstädtischer Wanderungsströme (Abb. 4), während viele - nicht generell alle! Großwohnsiedlungen wie auch einfache gründerzeitliche Quartiere ausgezehrt wurden. Bereits Ende der 1990er Jahre war somit in den ostdeutschen Großstädten - nach der Westwanderung und der Stadt-UmlandWanderung - eine dritte große Welle räumlicher Bevölkerungsmobilität zu beobachten: der Zuzug in die gehobenen innenstadtnahen Wohnungsteilmärkte. Somit hat auch in den Kernstädten wieder ein räumlicher Konzentrationsprozess eingesetzt, wenngleich dadurch Bevölkerungsdichten, wie sie vor der Wende bestanden, nicht wieder erreicht werden.

\section{Abbildung 4}

Saldierte Wanderungsströme in das gründerzeitliche Wohnquartier Leipzig-Gohlis-Süd 2000

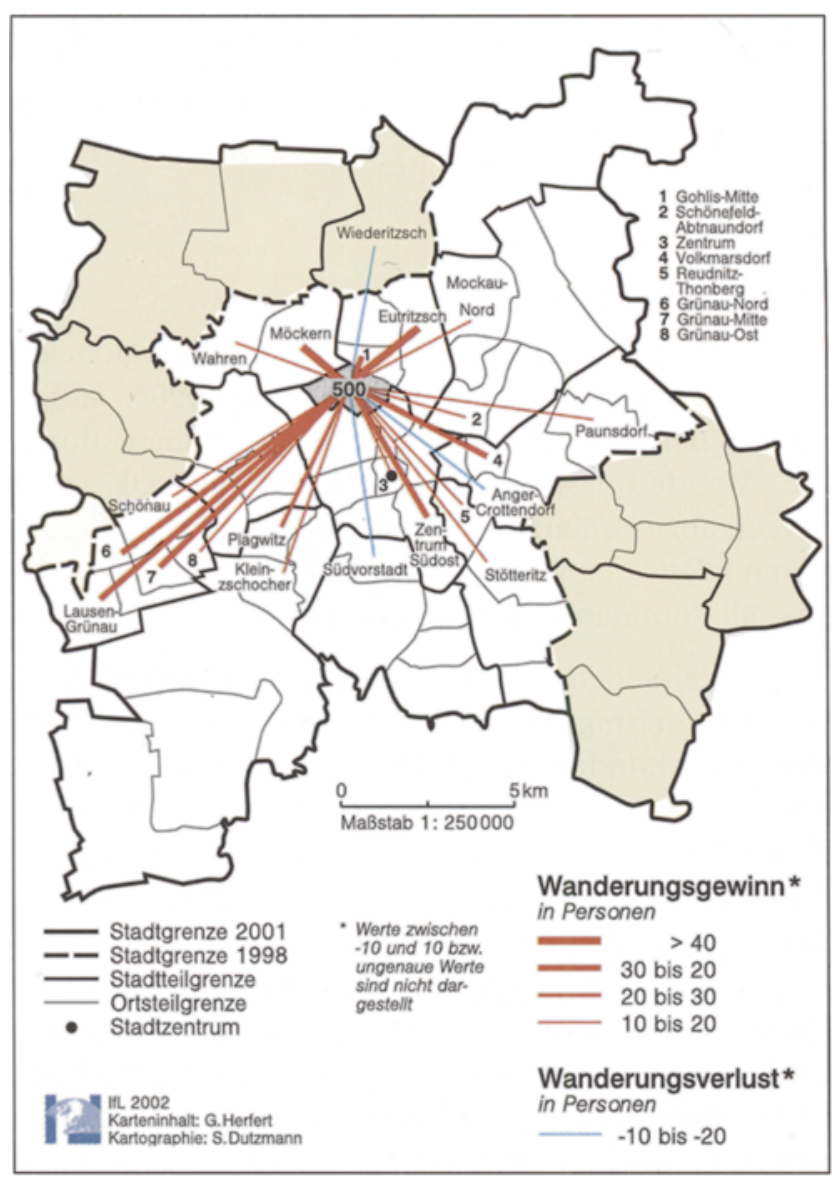

Ein Effekt der 1990er-Förderpolitik ,in der Fläche“ war die geringe interregionale Differenzierung der Bevölkerungsentwicklung in Ostdeutschland, da u.a. über die Wohnsuburbanisierung bedeutende Bevölkerungspotenziale in den Regionen gebunden wurden. Mit dem rapiden Rückgang der Stadt-Umland-Wanderung zum Ende der 90er Jahre, dem sich zunehmend differenzierenden regionalen Arbeits-/Ausbildungsplatzangebot und der steigenden Arbeitslosigkeit setzte nun jene Raumentwicklung ein, wie sie bereits nach der Wende für die neuen Länder prognostiziert wurde (BfLR 1993): die Ausprägung polarisierter Entwick-* lungsmuster mit stabilen bis leicht wachsenden Stadtregionen auf der einen und mehr oder weniger schrumpfenden Stadtregionen auf der anderen Seite.

\section{Polarisierung der Bevölkerungsentwicklung}

Der Trend zur räumlichen Polarisierung der Bevölkerungsentwicklung zeigt sich Anfang des neuen Jahrhunderts auch zwischen den Regionstypen, den Agglomerationsräumen einerseits und den verstädterten Räumen sowie ländlichen Räumen andererseits (Tab. 1) - selbst bei großen Streuungsbreiten innerhalb der Raumkategorien. Bei weiterer Untergliederung nach siedlungsstrukturellen Kreistypen (Abb. 5) differenziert sich das Bild weiter: Die höchsten Bevölkerungsverluste hatten danach die Kernstädte und verdichteten Kreise in den verstädterten Räumen sowie die ländlichen Räume zu verzeichnen - weniger davon betroffen waren die ländlichen Kreise der anderen Raumkategorien. Die eigentlichen Verlierer der aktuellen Bevölkerungsentwicklung sind jedoch insbesondere die Klein- und Mittelstädte (10 000-50 000 Einwohner), und dies mit steigendem Trend.

Sachsen / Sachsen-Anhalt / Thüringen

Durchschnittliche jährliche Bevölkerungsentwicklung in Regionstypen 1990-2000

\begin{tabular}{|l|c|c|c|}
\hline \multirow{2}{*}{ Regionstyp } & \multicolumn{3}{|c|}{ Bevölkerungsentwicklung/Jahr in \% } \\
\cline { 2 - 4 } & $\mathbf{1 9 9 0 - 1 9 9 4}$ & $\mathbf{1 9 9 4 - 1 9 9 8}$ & $\mathbf{1 9 9 8 - 2 0 0 0}$ \\
\hline Agglomerationsraum & $-0,94$ & $-0,48$ & $-0,60$ \\
Verstädterter Raum & $-0,99$ & $-0,61$ & $-0,95$ \\
Ländlicher Raum & $-0,78$ & $-0,68$ & $-0,95$ \\
\hline
\end{tabular}

Quelle: Statistische Landesämter, eigene Berechnungen

Quelle: Herfert 2002 
Abbildung 5

Bevölkerungsentwicklung nach

siedlungsstrukturellen Kreistypen in Sachsen, Sachsen-Anhalt und Thüringen 1998-2000

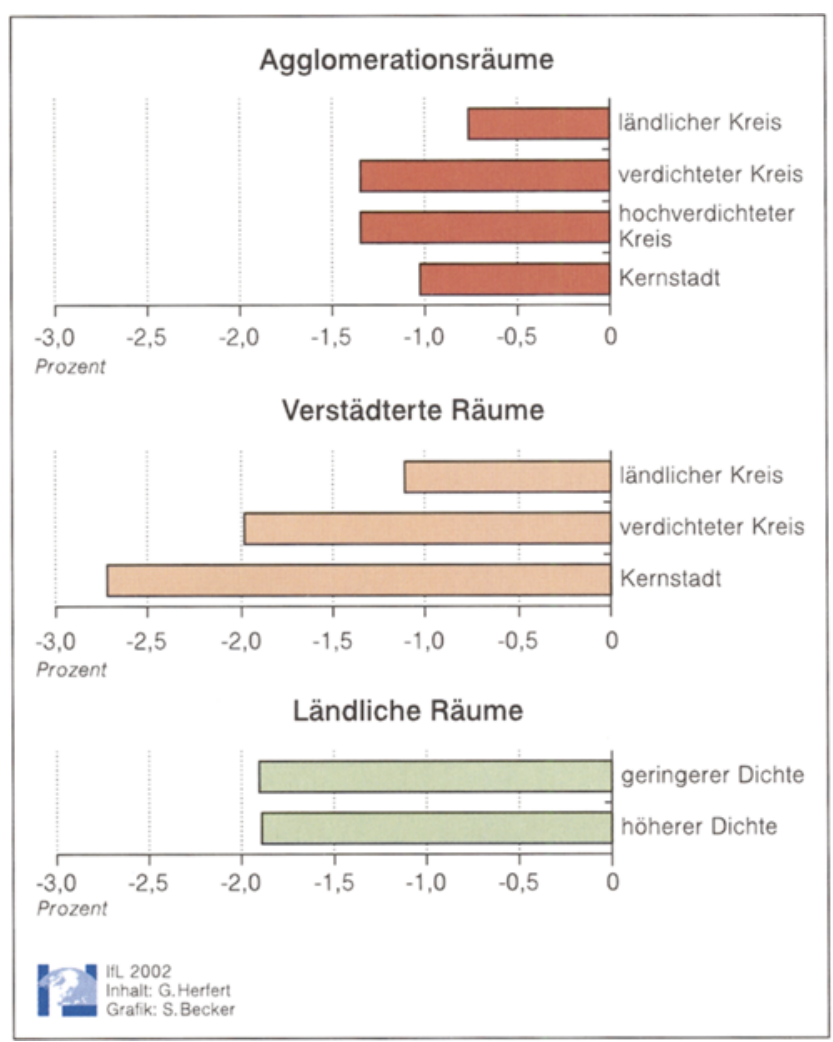

Quelle: Statistische Landesämter, eigene Berechnungen

\section{Abbildung 6}

Bevölkerungsentwicklung der Städte $>20000$ Einwohner in Sachsen, Sachsen-Anhalt und Thüringen 1998-2000

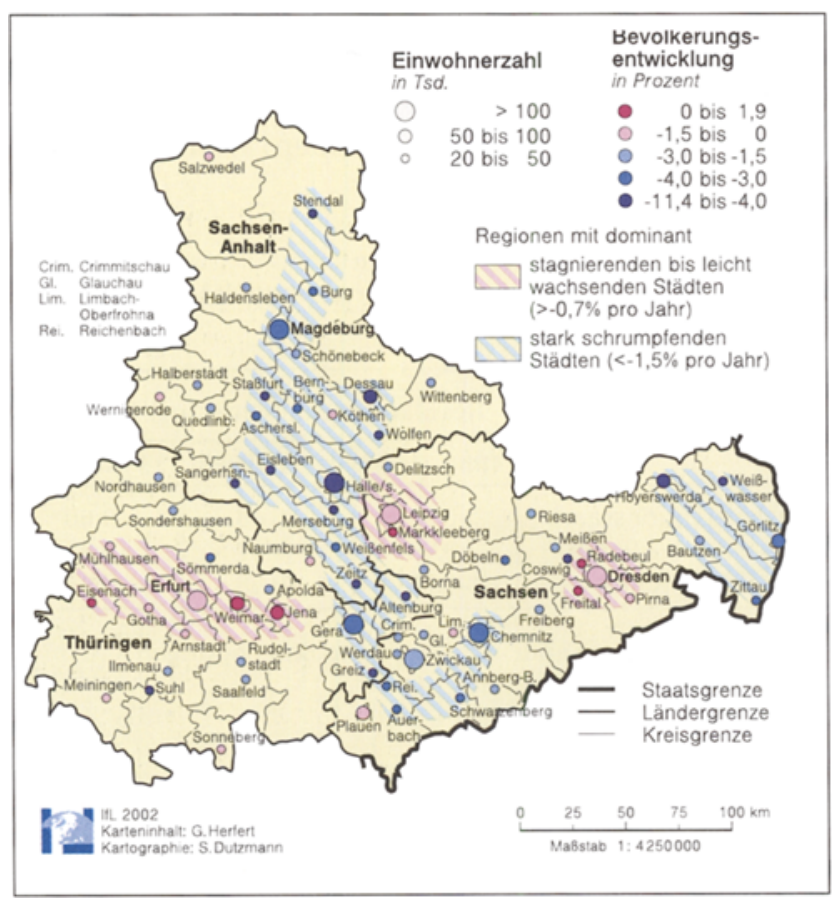

Quelle: Statistische Landesämter, eigene Berechnungen
Aber selbst diese Untergliederung trifft nicht den vollen Kern der aktuellen Polarisierungstendenzen: Es zeichnet sich vielmehr ein Bild von kleineren und größeren Inseln mit stabilen bis leicht wachsepden Bevölkerungspotenzialen $^{5}$ im ansonsten demographisch schrumpfenden Raum ab (Abb. 6). Da die wirtschaftliche Entwicklung der Gesellschaft heute vor allem von der Leistungsfähigkeit der Zentren bestimmt wird (Fürst 2001), insbesondere die Städte ab 20000 Einwohner als Träger größerer Potenziale die Regionalentwicklung wesentlich beeinflussen, kann man im Untersuchungsraum drei Regionstypen unterscheiden:

1. Regionen mit dominant stagnierenden bis leicht positiven Entwicklungen: die Stadtregionen Leipzig und Dresden sowie die thüringische Städtereihe von Jena bis Eisenach.

2. Das negative Pendant dazu mit überdurchschnittlichen Bevölkerungsverlusten: ein Band vorwiegend altindustrieller Regionen, das sich von Stendal durch den Kernraum Sachsen-Anhalts (Magdeburg/Dessau / Halle) über Ostthüringen (Gera, Greiz) bis in den sächsischen Verdichtungsraum Chemnitz erstreckt. Eine weitere Problemzone zeichnet sich in Ostsachsen mit dem oberzentralen Städteverbund Hoyerswerda-Bautzen-Görlitz ab.

3. Der restliche schrumpfende Raum: Regionen aller Raumtypen mit vorwiegend durchschnittlichen Bevölkerungsverlusten.

\section{Neue Wanderungsmuster}

Die Differenzierung der intra- als auch interregionalen Bevölkerungsentwicklung wird seit den 1990er Jahren infolge der ubiquitären Sterbefallüberschüsse durch die Wanderungskomponente bestimmt. Die sich derzeit abzeichnenden Polarisierungstendenzen resultieren folglich im Wesentlichen aus einem Wandel bzw. Wegfall dominanter Wanderungsströme.

Wie einleitend bereits erwähnt, läuft die Stadt-Umland-Wanderung in den Großstadtregionen Anfang des neuen Jahrhunderts im Saldo gegen Null ${ }^{6}$ bzw. es überwiegt bereits eine Umland-Stadt-Wanderung. Auch bei vielen Klein- und Mittelstädten sind wieder zentripetale Wanderungsströme dominant. In Westsachsen sind die Ansätze zur kleinräumigen Konzentration, also einer Reurbanisierung, fast flächendeckend (Abb. 7). Diese Entwicklung bereits als ein „Zurück-in-dieStadt" zu interpretieren, wäre jedoch überzogen. Haushaltsbefragungen in sächsischen Großstadtregionen (Wiest 2001) ergaben, dass z.B. von dem überdurchschnittlich hohen Anteil ( $>50 \%$ ) umzugswilliger Haus- 
Abbildung ?

Kleinräumige Reurbanisierungstendenzen in Westsachsen 1999-2000

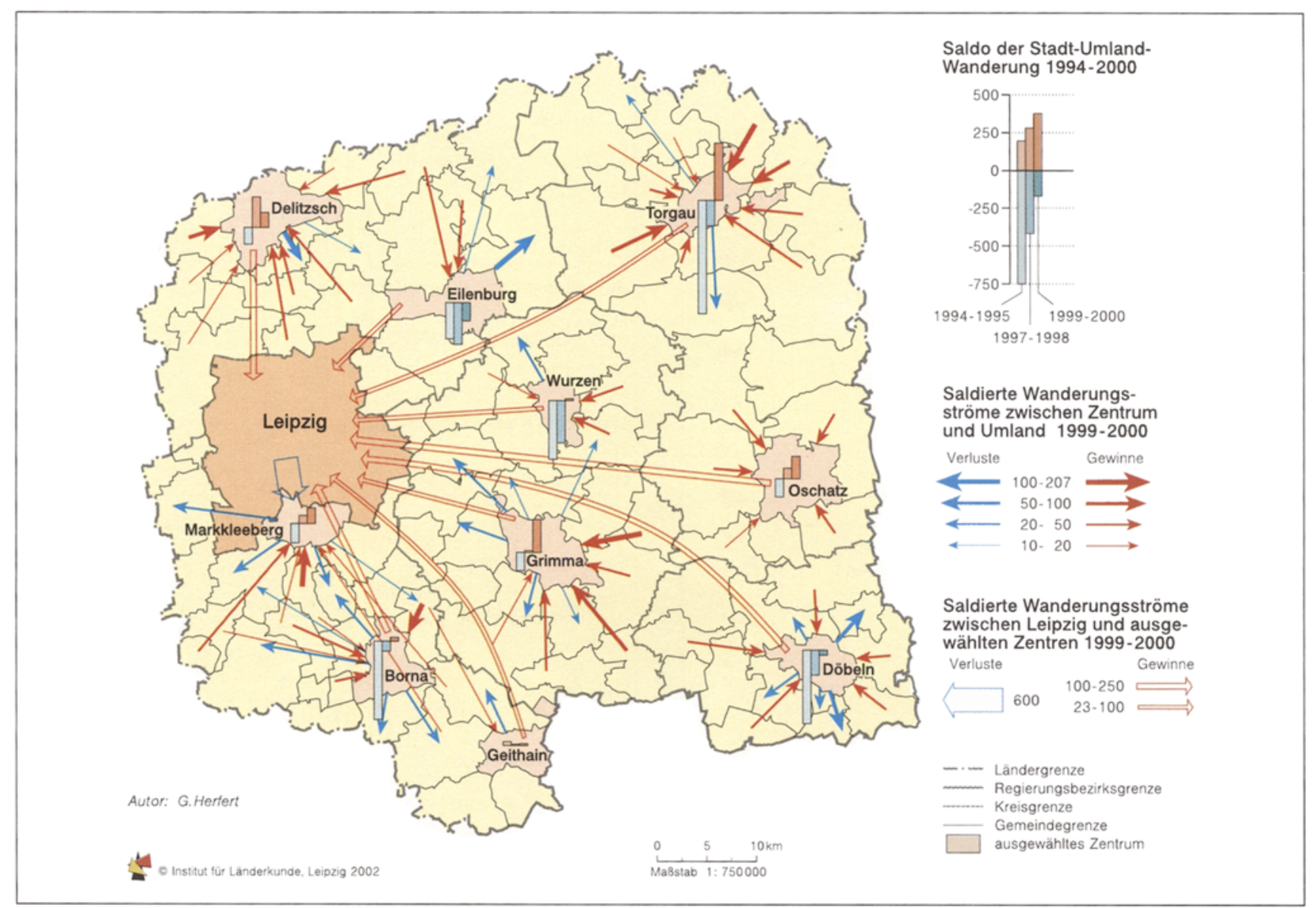

Quelle: Statistische Landesämter, eigene Berechnungen

halte in den neuen suburbanen Wohnparks vorerst nur wenige beabsichtigen, in die Kernstadt zurückzuziehen, eher näher an den Kernstadtrand. Zudem sind die neuen Eigenheimbesitzer durch Kredite an ihr Eigentum gebunden. Vorerst sind es vorrangig junge Bevölkerungsgruppen, die im Rahmen der Ausbildung bzw. Arbeitsaufnahme aus den kleinen Gemeinden in die Mittel- und Oberzentren abwandern -Trends, die im Rahmen des Lebenszyklus erfolgen. Dennoch gibt es zugleich Anzeichen für eine Neuorientierung auf dem Wohnungsmarkt. Denn auch im einst so dynamisch wachsenden suburbanen Raum sind zunehmend Wohnungsleerstände im Neu- und Altbaubestand sowie Insolvenzen im Einfamilienhausmarkt zu beobachten, liegt der Wiederverkaufswert der Wohnimmobilien bereits unter dem Neuwert, sind erste Haushalte unter dem Blickwinkel der wachsenden suburbanen Infrastrukturkosten, des sich abzeichnenden zunehmenden Wertverlustes ihrer Wohnimmobilien und der einsetzenden demographischen Schrumpfung in den wieder an Attraktivität gewinnenden städtischen Wohnungs- markt zurückgewandert. Dieser Trend dürfte sich bei Internalisierung der Kosten für das Wohnen „im Grünen“ weiter verstärken.

Wie ordnen sich nun diese kleinräumigen Konzentrationsprozesse in die neuen Wanderungsmuster ein und welche Rolle spielt dabei die - in der Öffentlichkeit so hochgespielte - neue Abwanderungswelle in die alten Länder?

Die Wanderungsbilanzen mit den alten Ländern sind gegenwärtig in den Oberzentren bei generell stark rückläufiger Stadt-Umland-Wanderung wieder zur entscheidenden Komponente der sich polarisierenden Bevölkerungsentwicklung geworden (Abb. 8). Diese Ost-West-Wanderung ist jedoch nicht vorrangig - wie vielfach vermutet - durch Einkommensdifferenzen determiniert, sondern durch die unterschiedliche Arbeitsmarktsituation in den neuen Ländern, insbesondere jedoch durch die mikroökonomischen Determinanten $^{7}$ (Dreger 2002). Auch wenn im Vergleich zur Wende in wesentlich geringeren Dimensio- 


\section{Abbildung 8}

Wanderungsmuster von Oberzentren in Sachsen, Sachsen-Anhalt und Thüringen 1998-2000

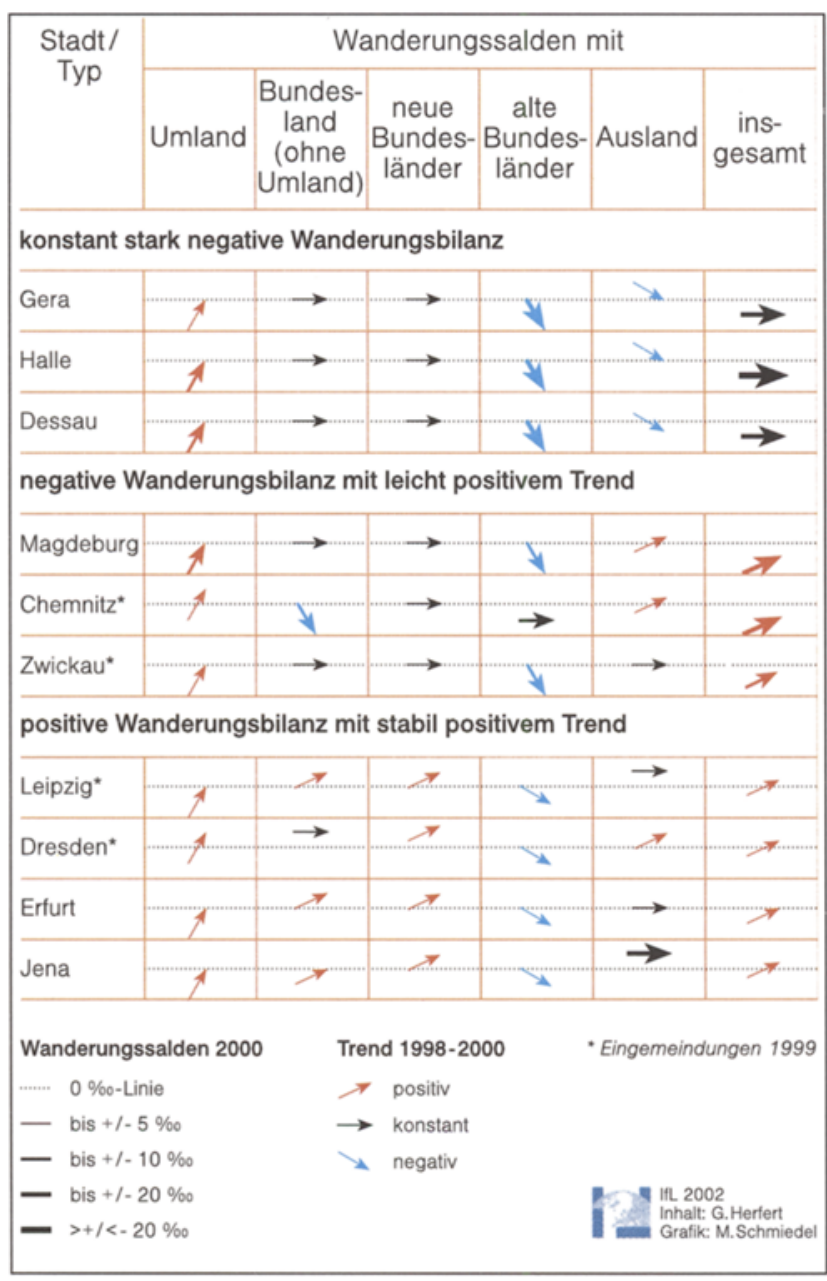

Quelle: Statistische Landesämter, eigene Berechnungen

nen, zeigt sich heute im Ergebnis dieser Entwicklung ein differenzierteres Raummuster. So haben die „Stabilitätsinseln“ Leipzig, Dresden, Erfurt und Jena auf Grund ihrer Arbeits-/Ausbildungsplatzattraktivität nur sehr geringe Wanderungsverluste Richtung alte Länder. Da zudem der Trend aus anderen Regionen sogar leicht positiv ist - was im Osten Deutschlands nur für die Stabilitätsinseln typisch ist -, zeigt sich hier seit 1998 ein stabil positiver Wachstumstrend. Im Gegensatz dazu haben die Oberzentren Gera, Halle und Dessau ihre insgesamt stark negative Wanderungsbilanz trotz rückläufiger Stadt-Umland-Wanderung beibehalten, in erster Linie verursacht durch die deutlich gestiegenen Westwanderungen. Mit jährlichen Wanderungsverlusten von bis $\mathrm{zu} 20 \%$ gehören diese Oberzentren - schon auf Grund ihres quantitativen Potenzials - zu den Hauptquellgebieten der aktuellen Ost-West-Wanderung. Stabilisierende Wanderungsströme aus anderen Regionen fehlen hier völlig. Magdeburg, Chemnitz

\section{Abbildung 9}

Wanderungsmuster ausgewählter Siedlungsstrukturtypen in Sachsen, Sachsen-Anhalt und Thüringen 1998-2000

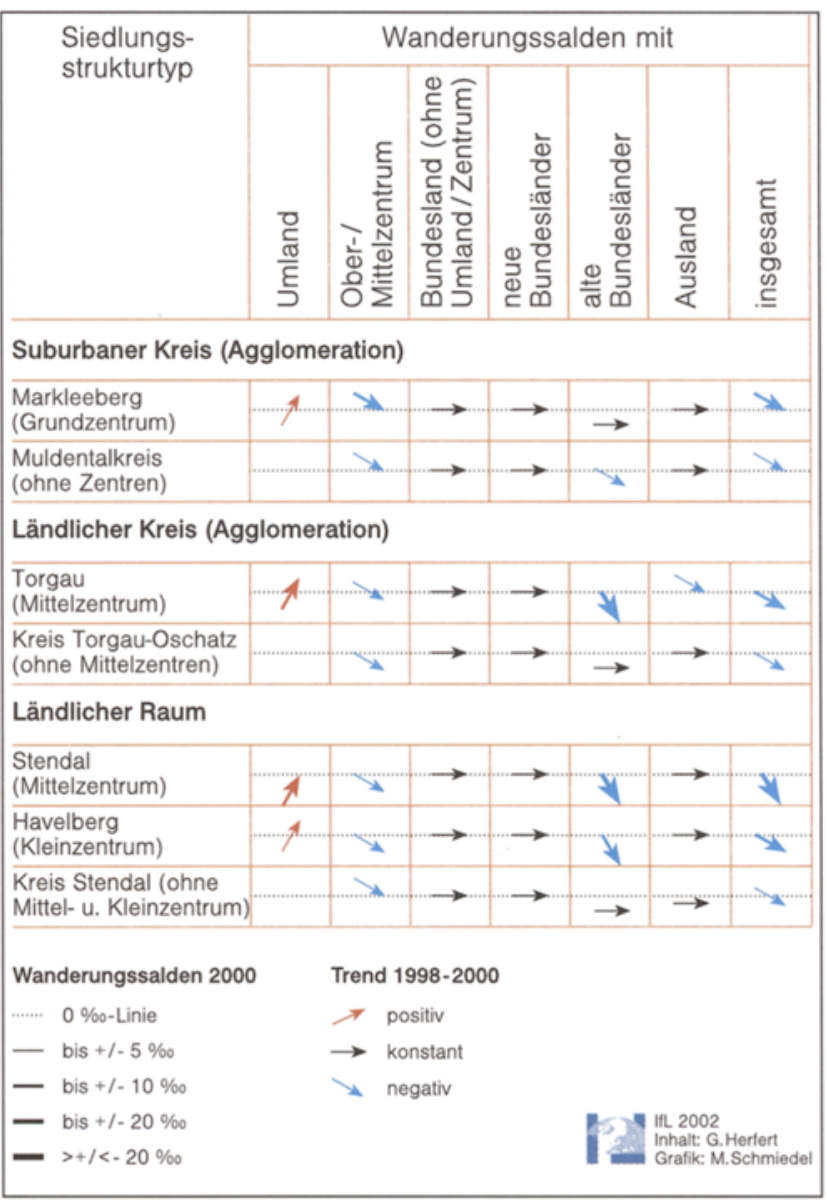

Quelle: Statistische Landesämter, eigene Berechnungen

und vor allem Zwickau zeigen Ansätze, sich aus diesem Negativ-Trend herauszulösen. Auffallend und zugleich auch typisch für die aktuelle und zukünftige Entwicklung in Ostdeutschland dürfte das unmittelbare Nebeneinander unterschiedlicher Entwicklungstrends sein, wie sie sich im Verdichtungsraum Halle-Leipzig manifestieren.

In den anderen Zentren- und Regionstypen waren Anfang des neuen Jahrhunderts generell nur negative Wanderungstrends zu verzeichnen, von deutlich rückläufigen Wanderungsgewinnen in den suburbanen Räumen bis hin zu extrem wachsenden Wanderungsverlusten in den Mittel- und Kleinzentren (Abb. 9). Letztere sind - wie auch die Mehrzahl der Oberzentren - vor allem von der Westwanderung betroffen, die die kleinräumigen Konzentrationsprozesse überlagert. Wenn auch noch quantitativ wesentlich geringer als zu Beginn der 90er Jahre, dürften die Verluste an Human- 
kapital ( -10 bis $-20 \%$ pro Jahr) qualitativ beträchtlich sein. Im Vergleich dazu sind die Wanderungsverluste in den kleineren Gemeinden (<2000 Einw.) der ländlichen Kreise eher gering geblieben. Beispielhaft dafür ist der Kreis Stendal, ein ländlicher Kreis im Norden Sachsen-Anhalts, der zu den strukturschwächsten Räumen Ostdeutschlands zählt (s. Abb. 9). Auch hier ist in der Kreisstadt (39795 Einw.: 2000) als auch im Grundzentrum Havelberg (ehemalige Kreisstadt mit 6451 Einw.: 2000) ein deutlicher Rückgang der StadtUmland-Wanderung zu verzeichnen, gleichzeitig ein fast explosiver Anstieg der Westwanderung im Jahre 2000. Stendal erreichte so im Gesamt-Wanderungssaldo fast die -30\%o-Grenze, die Kleinstädte des Kreises hatten ebenfalls deutliche Verluste $\mathrm{zu}$ verzeichnen. Im restlichen Kreisgebiet (Gemeinden $<5000$ Einwohner ohne Umland von Stendal) war die Wanderungsbilanz hingegen fast ausgeglichen. Der Faktor Wanderung tritt hier als Komponente der Bevölkerungsentwicklung in den Hintergrund, da auch die Westwanderung nur gering ausgeprägt ist. Diese scheinbare demographische Stabilität ist zum einen bedingt durch fehlende Abwanderungspotenziale - bereits vor der Wende waren diese Räume durch Schrumpfung und Überalterung geprägt - und zum anderen durch erzwungene (Immobilienbesitz) und gewollte (Identifikation mit der Region) Immobilität. Letztlich läuft hier seit der Wende ein überwiegend biologisch geprägter Schrumpfungsprozess, verbunden mit gravierenden Überalterungstendenzen. Da diese Bevölkerungsverluste jedoch geringer sind als in den Zentren, setzt sich im Kreis Stendal trotz rückläufiger Stadt-Umland-Wanderung die räumliche Dekonzentration der Bevölkerung fort.

\section{Trendwende zur Reurbanisierung?}

Dieser Trend, wie er sich im Kreis Stendal zeigt, beschränkt sich nicht nur auf den ländlichen Raum, er ist vielmehr Anfang des Jahrhunderts ein in Ostdeutschland weit verbreitetes Phänomen. Nach einer Phase der absoluten Dekonzentration in den Stadtregionen im Rahmen des Suburbanisierungsprozesses - mit deutlichem Bevölkerungszuwachs in den suburbanen Räumen - hat nun eine relative Dekonzentrationsphase eingesetzt (Abb. 10), ein großräumiger Disurbanisierungsprozess ${ }^{8}$, der sich - bei generell schrumpfenden Bevölkerungspotenzialen - über alle Typen von Raumstrukturen erstreckt. Zeitgleich auftretende kleinräumige Konzentrationsprozesse werden dabei von starken interregionalen Fortzügen überlagert. Ausgenommen von diesem fast flächendeckenden Disurbanisierungsprozess in Ostdeutschland sind die wenigen Stabilitätsinseln, jene Stadtregionen mit demographisch konstanten bis leicht wachsenden Kernstädten

\section{Abbildung 10}

Anteil der Bevölkerung außerhalb von Ober- und Mittelzentren in ausgewählten Regionen Sachsens, Sachsen-Anhalts und Thüringens 1990-2000

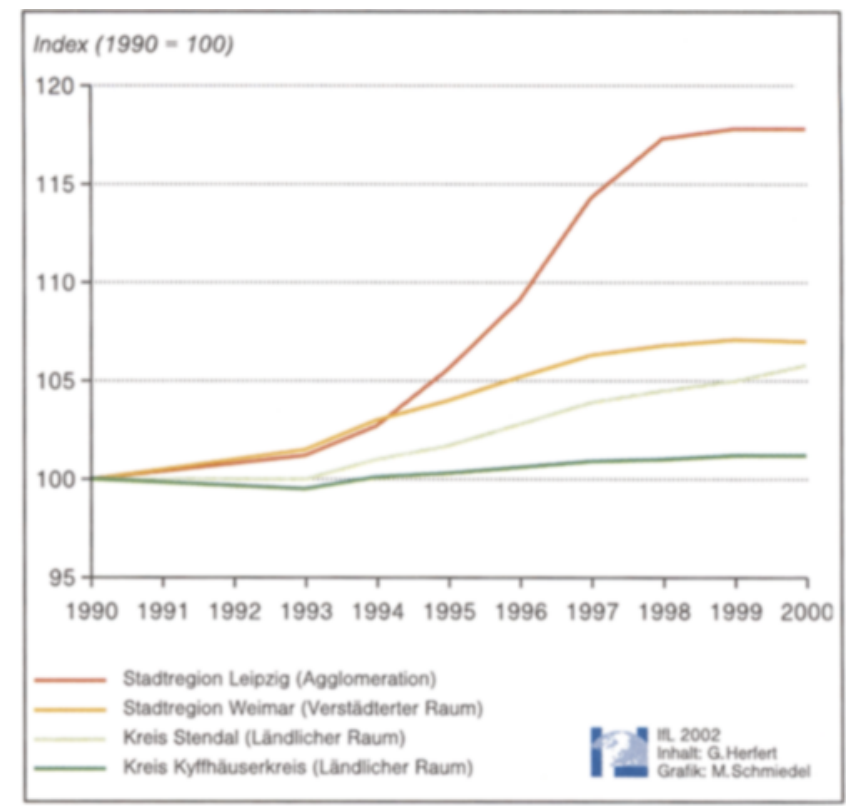

Quelle: Statistische Landesämter, eigene Berechnungen

einerseits und wieder leicht schrumpfenden suburbanen Räumen andererseits, wo Ansätze einer Reurbanisierung erkennbar sind. Zugleich polarisiert sich in diesen Stadtregionen der suburbane Raum: Während die Mehrzahl der kleinen suburbanen Gemeinden Bevölkerung verliert, bilden einige kernstadtnahe, verkehrsmäßig gut erschlossenen Kleinstädte Reststandorte des Wachstums. Dieses Wachstum wird gespeist von geringen, aber anhaltenden Zuwanderungen aus der Kernstadt, wobei nach Jahren vielfach „blinder" StadtUmland-Wanderung Lagepotenziale und Wohnumfeldbedingungen $\mathrm{zu}$ entscheidenden Faktoren der Wohnstandortwahl geworden sind. Diese Standortqualitäten sind es, die auch intraregionale Zuzüge aus dem suburbanen Umland in diese Kleinstädte auslösen. Es ist davon auszugehen, dass sich dieser Trend stabilisieren wird.

Den Negativpol zu diesen stabilen Kernen im suburbanen Raum stellen viele, zumeist peripher gelegene neue mehrgeschossige Wohnparks dar, die nicht nur auf Grund ihres hohen Wegzugspotenzials, sondern auch auf Grund des geringen natürlichen Wachstumspotenzials Anteil der Personen zwischen 19 und 35 Jahren < $30 \%$ ) zunehmend Wohnungsleerstandsprobleme aufweisen. Diese mehrgeschossigen Wohnsiedlungen sind neben vielen Platten- und einfachen gründerzeitlichen Siedlungen in den Kernstädten die aktuellen Problemquartiere der Stadtregionen (Wiest 2001). 


\section{Ausblick und Schlussfolgerungen}

Die Polarisierung der siedlungsstrukturellen Entwicklung spiegelt sich Anfang des neuen Jahrhunderts in Sachsen, Sachsen-Anhalt und Thüringen - letztlich trifft dies für den gesamten Osten Deutschlands zu in einer demographischen Schrumpfungslandschaft mit Stabilitätsinseln einerseits und räumlichen Clustern überdurchschnittlichen Bevölkerungsverlustes andererseits wider. Damit hat sich innerhalb eines Jahrzehnts der Trend der Bevölkerungsentwicklung in vielen Gemeinden grundlegend verändert. Der kleinräumigen Suburbanisierung folgte eine großräumige Disurbanisierung mit wenigen Reurbanisierungsinseln. Die empirische Realität zeigt somit ein Nebeneinander von gegensätzliche Entwicklungstendenzen der Stadt- und Regionalentwicklung (s.a. Läpple 2001) und ein Fehlen von eindeutigen Entwicklungsmustern.

Während in den alten Ländern konjunkturelle Schwankungen den anhaltenden Suburbanisierungssprozess zeitweilig deutlich abgeschwächt haben, ist in den neuen Ländern nach dem „Wegbrechen" der Wohnsuburbanisierung vorerst nicht mit einer Zyklizität der Bevölkerungs- und Raumentwicklung zu rechnen - der langfristige Schrumpfungsprozess ist vorgezeichnet. Szenarien der Westwanderung (Steinmann u. Tagge 2002) - basierend auf leichten Zuwanderungen aus dem Ausland und einer Ost-West-Anpassung der Fertilität in den neuen Ländern - erwarten bis 2050 in Ostdeutschland im negativsten Fall nur noch ein Potenzial von 8,6 Mio. Einwohnern, ohne Westwanderung wären es 13 Mio. Auf Grund der verstärkten Abwanderung erwerbstätiger Personen würde sich dann die Quote der Rentner pro 100 Erwerbstätige um 11,84 Prozentpunkte auf $58,6 \%$ erhöhen.

Infolge dieses langfristig prognostizierten Bevölkerungsrückganges ${ }^{9}$ und des daraus resultierenden fehlenden demographischen Wachstumsdrucks ist eine Wiederbelebung der Wohnsuburbanisierung in den neuen Ländern eher unwahrscheinlich - es sei denn, die Rahmenbedingungen ändern sich grundlegend. Die sich in Ansätzen zeigende Trendwende der Raumentwicklung dürfte deshalb - trotz des kurzen Untersuchungszeitraumes - zunehmend an Stabilität gewinnen.

Weitflächig ist somit im Osten Deutschlands, insbesondere auf Grund der neuen Wanderungstrends, ein anhaltender Disurbanisierungsprozess zu erwarten. Selbst in einer Stabilitätsinsel wie z. B. Leipzig, wo es mit dem Bau des BMW-Werkes zu einem leichten wirtschaftlichen Aufschwung in der Region kommen sollte und in diesem Zusammenhang vielfach auch über eine neue Suburbanisierungswelle spekuliert wird, ist eher ein Szenario „Reurbanisierung“ zu erwarten. Denn bereits heute ist voraussehbar, dass mit der rückläufigen Haushaltsentwicklung infolge des Geburtenlochs nach der Wende eine weitere Wohnungsleerstandswelle ab 2010 eintreten wird. Der einst so dynamische suburbane Raum wird dann nur noch für wenige, die sich zudem ohne Förderung und trotz zusätzlicher finanzieller Aufwendungen den Traum vom Eigenheim im Umland erfüllen wollen, Ziel möglicher Wünsche sein. Steigende Infrastruktur- und Transportkosten sowie die früher oder später reduzierte/wegfallende Eigentumsförderung für Neubauten - was angesichts der Wohnungsleerstände dringend notwendig wäre - als auch attraktive innerstädtische Wohnalternativen im Rahmen des Stadtumbaus Ost dürften hier eher zu einer Stabilisierung urbaner Strukturen führen.

Dieses Szenario gilt jedoch vorerst nur für die wenigen Stabilitätsinseln. In Regionen demographischer Schrumpfung, insbesondere dort, wo sich Städte mit hohen Bevölkerungsverlusten räumlich konzentrieren, zeichnet sich hingegen eine deutliche Schwächung des Netzes tragfähiger mittlerer Zentren (Müller $u$. Wichmann 2002) und damit eine Gefährdung nachhaltiger siedlungsstruktureller Entwicklungen ab. Gerade der Fakt, dass insbesondere die Städte - und nicht der ländliche bzw. altindustrielle Raum an sich - Verlierer der aktuellen demographischen Entwicklung sind, zwingt kurzfristig zu einer Profilierung der raumstrukturellen Leitidee des Zentrale-Orte-Konzeptes im Sinne einer Konzentration der Potenziale auf eine geringere Zahl Zentraler Orte (Danielzyk u. Winkel 2002) und zu einer prourbanen Förderpolitik, weg vom Gießkannenprinzip. Geht man davon aus, dass sich die Arbeitsmarktsituation kurzfristig nicht ändern wird, so muss man mit einem weiterhin verstärkten Abfluss von Humankapital aus den Städten rechnen. Deshalb ist es umso notwendiger, die aktuell kleinräumigen Konzentrationstendenzen im Siedlungsnetz, die regionalplanerisch jahrelang ohne Erfolg angestrebt wurden und sich nun als "natürliche" Tendenz anbieten, zu stärken. Neue Stadtentwicklungsprogramme, die im Rahmen des Förderprogramms "Stadtumbau Ost“ erstellt werden, sollten unter den sich abzeichnenden Entwicklungsbedingungen deutlich die Stadt als Standort zukünftig stabiler Lebensbedingungen proklamieren. Eine Pro-Stadt-Politik bedeutet nun nicht, den ländlichen (außerstädtischen) Raum zu vernachlässigen. Auch in peripheren Räumen sind finanzielle Anreize zur Sicherung des Humankapitals nötig. Aber angesichts des zu erwartenden starken Bevölkerungsrückgangs in Ostdeutschland dürfte das Ziel der Angleichung der Lebensbedingungen zwischen den Regionen illusorisch, da volkswirtschaftlich nicht realisierbar sein, wenn einschneidende Maßnahmen bis 
hin zur Auflösung von Siedlungsstrukturen nicht geplant und umgesetzt werden, um den Schrumpfungsprozess nachhaltig zu bewältigen. Das schließt ein, „die nach wie vor bestehende Tabuisierung der Schrumpfungsproblematik und vor allem der notwendigen Debatte um ihre politischen Schlussfolgerungen aufzubrechen“ (Müller u. Wichmann 2002).

\section{Anmerkungen}

* Der Beitrag wurde im Rahmen der ARL-Arbeitsgruppe „Regionalentwicklung unter Schrumpfungsbedingungen" erarbeitet.

(1)

Siedentop (2002) kommt im Rahmen eines Ost-West-Vergleichs deutscher Stadtregionen infolge einer sehr weiträumigen Abgrenzung der Stadtregionen (60-km-Radius) zu dem Ergebnis, dass für die siedlungsstrukturelle Entwicklung Ostdeutschlands nicht die klassische Suburbanisierung wie in den alten Ländern, sondern die Disurbanisierung charakteristisch ist. Zugleich verweist er aber auch darauf, dass der Terminus der Disurbanisierung in der Literatur durch die Verlagerung von Wachstumsschwerpunkten in Regionen außerhalb der Agglomerationsräume belegt ist. Diesem Kontext entspricht jedoch in keiner Weise die ostdeutsche Raumentwicklung der 1990er Jahre, die in Stadtregionen vielmehr als kleinräumiger Dekonzentrationsprozess - maximal in einem Radius von $15 \mathrm{~km}$ um die Kernstadt - unter generellen Schrumpfungsbedingungen erfolgte. Grundsätzlich ist in diesem Zusammenhang darauf zu verweisen, dass die Verwendung der Begriffssystematik von van den Berg und Klassen (1987) unter diesen Rahmenbedingungen problematisch ist. Darüber hinaus gibt es in der Literatur aber überwiegend Konsens hinsichtlich der Bewertung des Suburbanisierungsprozesses in Ostdeutschland als einer typischen Phase ostdeutscher Stadtregionsentwicklung in den 1990er Jahren nach weitestgehend eigenen Entwicklungsmustern.

(2)

Auch unter Berücksichtigung der umfangreichen Eingemeindungen nach Leipzig am 1.1.1999

(3)

Conrad (1980) bewertete die Stadt-Rand-Wanderung in den alten Ländern hinsichtlich der Zusatzausgaben der Randgemeinden für die Neubürger und der weitgehend konstanten Ausgaben der Kernstädte sowie der volkswirtschaftlichen Kosten für die vermehrte Mobilität als volkswirtschaftlich verschwenderisch (s.a. Mäding 2001).

(4)

Diese Gruppierungen überdecken die demographische Schrumpfung vieler kleiner Gemeinden in den peripheren Räumen.

(5)

Es sind weniger Wachstums-, sondern eher Stabilitätsinseln im ansonsten demographisch schrumpfenden Raum.
(6)

Vielfach vermutet man dahinter auch einen statistischen Effekt, da sich Studenten auf Grund von Vergünstigungen seitens der Universitätsstädte zunehmend mit Hauptwohnsitz am Studienort anmelden. Selbst wenn dieser Effekt in gewissem Grade zu berücksichtigen wäre, so ist doch gleichzeitig ein deutlich negativer Trend bei den Fortzügen aus den Kernstädten ins Umland zu beobachten.

(7)

Dazu zählt Dreger (2002) u.a. Alter, Familienstand, Bildungsniveau und individuelles Erwerbseinkommen. Er verweist darauf, dass Wanderungsbewegungen erst dann induziert werden, wenn der Nutzen des Wohnungswechsels die Schwelle der Transportkosten übersteigt.

(8)

Disurbanisierung ist hier als weitestgehend flächendeckendes Phänomen der ostdeutschen Raumentwicklung zu verstehen, nicht als Verlagerung von Wachstumsschwerpunkten in Regionen außerhalb der Agglomerationsräume.

(9)

Veränderungen der Fertilität und Alterung, die die Bevölkerungsentwicklung ebenfalls stark beeinflussen, wurden hier nicht explizit dargestellt.

\section{Literatur}

Aring, J.; Herfert, G. (2001): Neue Muster der Wohnsuburbanisierung. In: Brake, K.; Dangschat, J.S.; Herfert, G. (Hrsg.): Suburbanisierung in Deutschland. Aktuelle Tendenzen. S. 43-56

BfLR (1993): Mittel- und langfristige Entwicklungsperspektiven für deutsche Stadtregionen. = Materialien zur Raumentwicklung, H. 58

Conrad, H.-J. (1980): Stadt-Umland-Wanderung und Finanzwirtschaft der Kernstädte. - Frankfurt a. M.

Danielzyk, R.; Winkel, R. (2002): Auswirkungen der Schrumpfungsprozesse auf das raumordnerische Instrumentarium. In: Regionalentwicklung unter Schrumpfungsbedingungen. $=\mathrm{Ar}-$ beitsmaterial der ARL, Hannover (im Druck)

Dreger, Ch. (2002): Ost- und West-Migration in Deutschland kaum durch gesamtwirtschaftliche Entwicklung erklärbar. In: Wirtschaft im Wandel, H. 2, S. 47-50

Fürst, D. (2001): Einführung. Stadt und Region. In: Deutsche Zeitschrift für Kommunalwissenschaften, H. II, S. 5-12

Herfert, G. (1998): Stadt-Umland-Wanderungen in den 90er Jahren. In: Informationen zur Raumentwicklung, H. 11/12, S. 763776

Herfert, G. (2001): Stadt-Umland-Wanderungen nach 1990. In Institut für Länderkunde (Hrsg.): Nationalatlas Bundesrepublik Deutschland, Bd. 4: Bevölkerung, S. 116-119 
Herfert, G. (2002): Kleinräumige Wanderungsprozesse in Westsachsen - Trendwende zur Reurbanisierung? In: Stadt Leipzig (Hrsg.): Statistischer Quartalsbericht 2, S. 13-16

Herfert, G.; Schulz, M. (2002): Wohnsuburbanisierung in Verdichtungsräumen. In Institut für Länderkunde (Hrsg.): Nationalatlas Bundesrepublik Deutschland, Bd. 5: Dörfer und Städte, S. $124-127$

Läpple, D. (2001): Stadt und Region in Zeiten der Globalisierung und Digitalisierung. In: Deutsche Zeitschrift für Kommunalwissenschaften, H. II, S. 12-36

Mäding, H. (2001): Suburbanisierung und kommunale Finanzen. In: Brake, K., Dangschat, J.S.; Herfert, G. (Hrsg.): Suburbanisierung in Deutschland. Aktuelle Tendenzen. S. 109-120

Müller, B.; Wichmann, Th. (2002): Anforderungen an Steuerungsansätze der Stadt- und Regionalentwicklung unter Schrumpfungsbedingungen. In: Regionalentwicklung unter Schrumpfungsbedingungen. $=$ Arbeitsmaterial der ARL, Hannover (im Druck)
Siedentop, St. (2002): Siedlungsstrukturelle Trends in Agglomerationsräumen - ein Ost-West-Vergleich. In: Moser, P. ; Thiele, K. (Hrsg.): Entwicklung kleiner und mittlerer Zentren im suburbanen Raum - Einordnung des Mittelzentrums Schkeuditz. In: Stadtökologische Forschungen Nr. 32, UFZ-Berichr, Nr. 5, S. 524

Steinmann, G.; Tagge, S. (2002): Determinanten der Bevölkerungsentwicklung in Ost- und Westdeutschland. In: Wirtschaft im Wandel, H. 4, S. 91-99

Wiest, K. (2001): Instabile Wohnquartiere in schrumpfenden Stadtregionen Sachsens - eine Analyse charakteristischer Problemkonstellationen. In: Europa regional, H. 4, S. 192-203

Dr. Günter Herfert Institut für Länderkunde Schongauerstraße 9

04329 Leipzig

E-Mail: G_herfert@ifl-leipzig.de 